Reprinted from Journal of Applied Puysics, Vol. 35, No. 6, 1764-1767, June 1964

Copyright 1964 by the American Institute of Physics Printed in U. S. A.

\title{
Superposition of Semi-Infinite Solenoids for Calculating Magnetic Fields of Thick Solenoids
}

Gerald V. Brown and Lawrexce Flax

Lewis Research Center, National Aeronaulics and Space Administration, Cleveland, Ohio

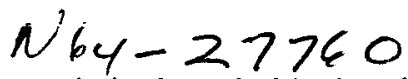

(Rectived 11 December 1963)

A simple method is given for calculating the magnetic field components in or around any thick finite solenoid by superposition of fields of semi-infinite solenoids with zero inner radius. Equations and graphs are presented for the field components of such semi-infinite solenoids. From these graphs, the fields for solenoids of practical interest can be obtained with errors of less than a few percent. Greater accuracy is possible if numerical tables are used instead of graphs.

\section{INTRODUCTION}

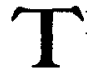
HE magnetic field components off the axis of a thick solenoid cannot be calculated easily except by electronic computers. Tables of these fields, prepared from computer output, would of ten be useful, especially in coil design. Such tables would eliminate further use of computers in many cases. But the field components of a thick solenoid depend on four variables, which are the radial and axial coordinates of the field point and the two parameters $\alpha$ and $\beta$ needed to specify the solenoid shape, where $\alpha=$ outer diameter/inner diameter and $\beta=$ length $/$ inner diameter. Tabulating this function of four continuous variables would require an unreasonable amount of space for complete coverage of the range of variables of common interest, although some tables and graphs exist which partially cover the ranges of the four variables. ${ }^{1-4}$

However, a comprehensive presentation in terms of only two variables is possible if a superposition method is used to find the desired field from tables or graphs of the field of a semi-infinite solenoid with zero inner radius. This semi-infinite solenoid is defined as an axially symmetric, uniform, azimuthal current density which extends from the axis of a cylindrical coordinate system out to $R=b$ and from $Z=0$ to $Z=-\infty$ (Fig. 1). It differs from ordinary solenoids in that it is semiinfinitely long and has no cylindrical hole. Each field component of this special solenoid can be expressed

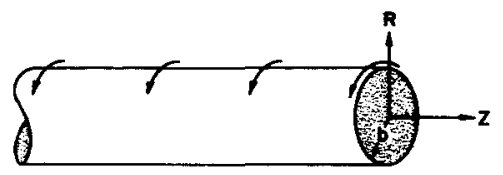

FIG. 1. Semi-infinite solenoid with zero inner radius. Current density is uniform, extending from axis to $R=b$ and from $Z=0$ to $Z=-\infty$. Curved arrows indicate direction of current circulation.

${ }^{1}$ G. V. Brown, L. Flax, E. C. Itean, and J. C. Laurence, "Axial and Radial Magnetic Fields of Thick, Finite-Length Solenoids," NASA TR R-170 (1963).

2E. E. Callaghan and S. H. Maslen, "The Magnetic Field of a Finite Solenoid," NASA TN D-465 (1960).

${ }^{3}$ N. B. Alexander and A. C. Downing, "Tables for a SemiInfinite Circular Current Sheet," ORNL-2828 (1959).

$1 D$. E. Morris, and R. A. Kilgore "The Magnetic Field on the Axis of Circular Cylindrical Coils," NASA TN D-1013 (1962). nondimensionally, computed electronically, and tabulated or graphed as a function of only two variables, the nondimensional field-point coordinates, $r=R / b$ and $z=Z / b$. Any desired finite solenoid is then treated as a superposition of four of these semi-infinite solenoids, and any field component is obtained by algebraic addition of four numbers derived from the tables or graphs, each number corresponding to the contribution of one semi-infinite solenoid.

\section{SUPERPOSITION OF FOUR SEMI-INFINITE SOLE- NOIDS TO FORM A FINITE SOLENOID WITH FINITE THICKNESS}

Figure 2 shows how four semi-infinite solenoids of appropriate sizes and locations can be superimposed to form a thick, finite solenoid. Curved arrows show the directions of circulation of the currents. Cancellation of oppositely directed currents occurs in many regions, leaving only the desired finite solenoid.

The field of the thick, finite solenoid can be expressed in terms of the fields of the four contributing semiinfinite solenoids in the following manner. Consider

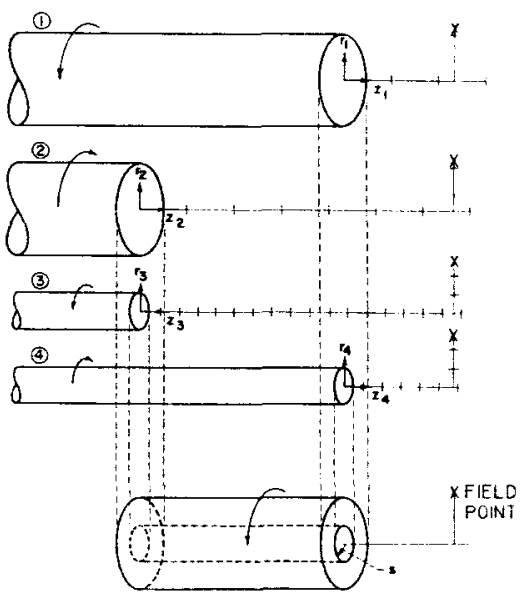

FIG. 2. Formation of finite solenoid from four semi-infinite solenoids. Curved arrows indicate directions of current circulation. Position of typical field point $(X)$ is shown with respect to the desired finite solenoid and with respect to each semi-infinite solenoid. Note that since nondimensional tables or graphs are to be used, each semi-infinite solenoid effectively has its own coordi nate system, with its own radius as the unit of length. 
either the axial or the radial component of the field of the infinite solenoid. To simplify notation, no symbols will be used to distinguish radial and axial field components; all equations in this section are valid for either component. Let $H_{1}, H_{2}, H_{3}$, and $H_{4}$ represent the values of the desired field component which would be contributed by the four semi-infinite solenoids marked 1 , 2,3 , and 4, respectively, in Fig. 2 if each had the conventional sense of current circulation. The actual fields contributed by the solenoids numbered 2 and 4 in the figure are thus $-H_{2}$ and $-H_{4}$, because the current densities have a reversed sense in these two semiinfinite solenoids. The field of the desired thick finite solenoid is simply the sum of the contributions of the four semi-infinite solenoids:

$$
H=H_{1}-H_{2}+H_{3}-H_{4} \text {. }
$$

If each term is divided by the current density $J$ (assumed to be uniform) and by the inner radius $s$ of the finite solenoid, a nondimensional expression results:

$$
H / J_{s}=H_{1} / J_{s}-H_{2} / J_{s}+H_{3} / J_{s}-H_{4} / J_{s} .
$$

Let the radii of the semi-infinite solenoids be called $b_{1}, b_{2}, b_{3}$, and $b_{4}$. Note that $b_{3}=b_{4}=s$ and $b_{1}=b_{2}=\alpha s$. Then

$$
H / J s=\alpha H_{1} / J b_{1}-\alpha H_{2} / J b_{2}+H_{3} / J b_{3}-H_{4} / J b_{4} .
$$

Let the nondimensional quantities such as $H_{1} / J b_{1}$ be denoted by $h_{1}$, etc. Then

$$
H / J_{S}=\alpha h_{1}-\alpha h_{2}+h_{3}-h_{4} .
$$

The quantities $h_{1}, h_{2}, h_{3}$, and $h_{4}$ are the nondimensional

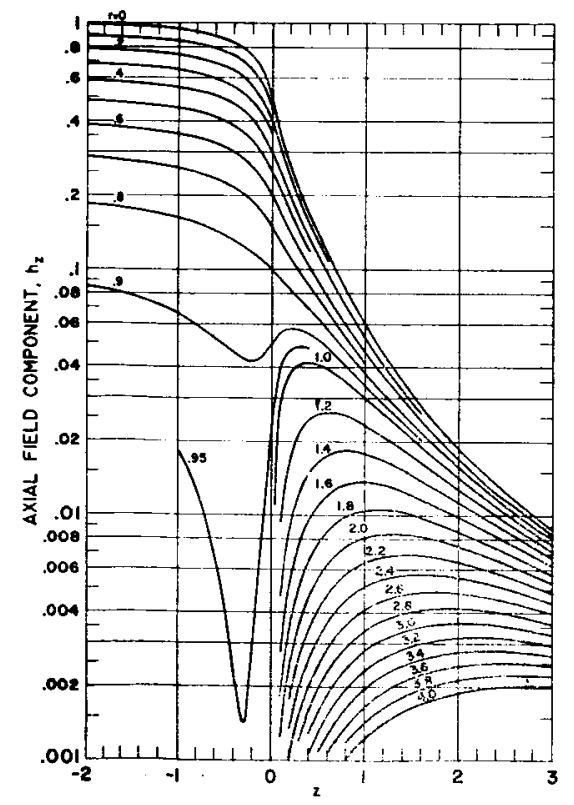

FIG. 3. Nondimensional axial field of semi-infinite solenoid. Note: For $z$ negative and $r \geq 1$, no curves are shown; for such values, use the relation: $h_{z}(r,-z)=-h_{z}(r, z)$ for $r \geq 1$.
Fig. 4. Nondimensional radial field of semi-infinite solenoirl. Note: Values for $z<0$ are not shown because $h_{r}(r,-z)=h_{r}(r, z)$.

fields of the four semi-infinite solenoids. These nondimensional fields can be approximately evaluated by using the graphs in Figs. 3 and 4 if the proper coordinate systems are used for locating the field point. The most convenient coordinate system to use with any particular semi-infinite solenoid is a cylindrical one with the origin at the center of the end face. It is convenient to nondimensionalize the radial and axial coordinates by expressing them in terms of the radius of the semiinfinite solenoid as a unit of measurement. Giving each semi-infinite solenoid its own origin of coordinates and unit of measurement means that the field point will have different coordinates with respect to each of the four semi-infinite solenoids. Figure 2 shows the coordinate system to be used for each semi-infinite solenoid: $\left(r_{1}, z_{1}\right)$ for solenoid $1,\left(r_{2}, z_{2}\right)$ for solenoid 2 ; etc. Note that the unit of length for measuring $r_{1}$ and $z_{1}$ is $b_{1}$; for $r_{3}$ and $z_{3}$ it is $b_{3}$; etc. Making these field point coordinates explicit in Eq. (2) gives

$$
H / J s=\alpha h\left(r_{1}, z_{1}\right)-\alpha h\left(r_{2}, z_{2}\right)+h\left(r_{3}, z_{3}\right)-h\left(r_{4}, z_{4}\right) .
$$

Equation (3), containing only nondimensional terms which are expressed in terms of nondimensional coordinates, can be used to calculate the field components of any solenoid of finite length and thickness from $h_{r}(r, z)$ and $h_{z}(r, z)$. The functions $h_{r}(r, z)$ and $h_{z}(r, z)$ are the nondimensional radial and axial components of the magnetic field intensity of the semi-infinite solenoid, and their derivations are sketched at the end of the paper. They have been computed numerically and are plotted in Figs. 3 and 4.

An example should clarify the procedure for calculating a field by using Eq. (3) and the graphs. Suppose the axial field component is desired at the indicated field point for the finite solenoid shown in cross section in 


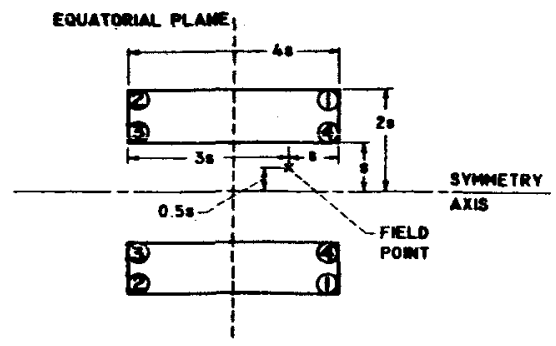

FIg. 5. Cross section of solenoid used in example calculation. Numbered corners correspond to locations of the edges of the end planes of the like-numbered semi-infinite solenoids of Fig. 2.

Fig. 5. The inner radius of this finite solenoid is s. Each corner in the cross section is numbered and indicates the location of the circular edge of the end plane of the semiinfinite solenoid with the same number in Fig. 2. This numbering also corresponds to that of Eq. (3). Comparing Figs. 2 and 5 , one can see that $r_{1}=0.5 / 2=0.25$, $r_{2}=0.5 / 2=0.25, \quad r_{3}=0.5, \quad r_{4}=0.5, \quad z_{1}=-\frac{1}{2}=-0.5$, $z_{2}=\frac{3}{2}=1.5, z_{3}=3$, and $z_{4}=-1$. Since $\alpha=2$, Eq. (3) becomes

$$
\begin{aligned}
H_{z /} / J_{s}=2 h_{z}(0.25,-0.5)-2 h_{z}(0.25,1.5) \\
+
\end{aligned}
$$

Figure 3 yields the necessary values of $h_{z}$, hence

$$
H_{z} / J_{s}=2 \times 0.62-2 \times 0.030+0.008-0.45=0.74 .
$$

Although $H / J s$ is a nondimensional quantity, the expressions used in calculating $h_{r}(r, z)$ and $h_{i}(r, z)$ were derived by using rationalized $m k s$ units, so a rationalized system of units must be used in these computations. Supposing $J=10^{7} \mathrm{~A} / \mathrm{m}^{2}$ and $s=0.05 \mathrm{~m}$ and noting that $B=\mu_{0} H$ in rationalized mks units results in the following for the axial component of magnetic induction:

$B_{z}=\mu_{0} J_{s} \times 0.74=4 \pi \times 10^{-7} \times 10^{7} \times 0.05 \times 0.74=0.46$ $\mathrm{Vb} / \mathrm{m}^{2}$, or $4600 \mathrm{G}$.

Reference 1 has a table of the fields of this particular solenoid and gives the more exact value of 0.7405 for $H_{z} / J s$. The error in the above result is fortuitously much less than $1 \%$, but errors for other solenoids that are neither too short nor too thin will be typically only a few percent if the graphs presented herein are used. However, for very short solenoids or for very thin ones, two terms in Eq. (3) may be nearly equal but opposite in sign, causing a loss of one or more leading digits through subtraction. At the end of a calculation, it can easily be seen whether accuracy has been lost in this way.

For calculating fields of such short or thin solenoids or to get greater accuracy for any solenoid, a tabulation of the field components of the semi-infinite solenoid is needed rather than graphs. Five-place tables and larger reproductions of Figs. 3 and 4 are to be published. However, approximate field values can be obtained from Figs. 3 and 4 for a very wide range of the shape parameters $\alpha$ and $\beta$. A remarkable feature of this method is that so much information about solenoids can be presented on these graphs and can be handled without further use of a computer.

The field of the semi-infinite solenoid has several properties which may be useful in calculations and some of which have been used to make Figs. 3 and 4 more compact. These are:

(1) For $z=0$ the nondimensional axial field component $h_{z}$ varies linearly from the value 0.5 on the axis to zero at $r=1$. For $z=0$ and $r \geq 1$ the axial field is zero.

(2) For a given value of $r$, the sum of the nondimensional axial field components at plus $z$ and at minus $z$ gives twice the value for $z=0$ at the same $r$ : $h_{z}(r, z)+h_{z}(r,-z)=2 h_{z}(r, 0)$.

(3) For $r \geq 1$ the nondimensional axial fields at positive and negative values of $z$ for a given radius are equal in magnitude but opposite in sign : $h_{z}(r, z)=-h_{z}(r,-z)$ for $r \geq 1$.

(4) For any given $r$ the nondimensional radial field components are equal both in magnitude and in sign for positive and negative $z: h_{r}(r, z)=h_{r}(r,-z)$.

\section{CALCULATION OF THE FIELD COMPONENTS OF THE SEMI-INFINITE SOLENOID}

Let $R, Z$, and $\Phi$ be the radial, axial, and azimuthal coordinates of the field point, as in Fig. 1, and let $a, l$, and $\theta$ be the coordinates of the source point. The origin of the coordinate system lies at the center of the end plane of the semi-infinite solenoid. The azimuthal coordinates $\theta=0$ and $\Phi=0$ are taken as coincident. The only nonvanishing component of the magnetic vector potential is the azimuthal component $A_{\Phi}$, which in rationalized mks units is

$$
A_{\Phi}=\frac{\mu_{0} J}{2 \pi} \int_{-\infty}^{0} d l \int_{0}^{b} d a \int_{0}^{\pi} \frac{a \cos \theta d \theta}{\left[(Z-l)^{2}+R^{2}+a^{2}-2 a R \cos \theta\right]^{]^{2}}} .
$$

Then by using $\mathbf{H}=\left(1 / \mu_{0}\right)$ curl $\mathbf{A}$, expressions for $H_{z}$ and $H_{\tau}$ can be obtained. Let $\xi=Z-l$, then

and

$$
H_{r}=\frac{J}{2 \pi} \int_{0}^{b} d a \int_{0}^{\pi} \frac{a \cos \theta d \theta}{\left[Z^{2}+R^{2}+a^{2}-2 a R \cos \theta\right]^{!}},
$$

$$
H_{z}=\frac{J}{2 \pi} \int_{Z}^{\infty} d \xi \int_{0}^{b} d a \int_{0}^{\pi} \frac{a \cos \theta d \theta}{\left[\xi^{2}+R^{2}+a^{2}-2 a R \cos \theta\right]^{i}} .
$$

The expression for $H_{r}$ can be integrated analytically with respect to $a$, and the expression for $H_{z}$ can be integrated analytically with respect to both $\xi$ and $a$. If the resulting expressions are divided by $J b$ and if the nondimensional field point variables $r=R / b$ and $z=Z / b$ are substituted into the expressions, the following results:

$$
\begin{aligned}
h_{r}(r, z) \equiv \frac{H_{\tau}}{J b}=\frac{1}{2 \pi} & \int_{0}^{\pi} F \cos \theta d \theta \\
& \quad+\frac{1}{2 \pi} \int_{0}^{\pi} r \cos ^{2} \theta \sinh ^{-1}\left[\frac{1-r \cos \theta}{\left(z^{2}+r^{2} \sin ^{2} \theta\right)^{\frac{1}{2}}}\right] d \theta
\end{aligned}
$$


1767 METHOD FOR CALCULATING MAGNETIC FIELDS OF SOLENOIDS

$$
\begin{aligned}
h_{z}(r, z) \equiv & \frac{H_{z}}{J b}=\frac{1}{2 \pi}\left\{-z \int_{0}^{\pi} \ln \left[\frac{2(1-r \cos \theta+F)}{|z|+\left(z^{2}+r^{2}\right)^{\frac{1}{2}}}\right] d \theta\right. \\
& +z r^{2} \int_{0}^{\pi} \frac{\sin ^{2} \theta d \theta}{\left(1+r^{2}-2 r \cos \theta\right) F} \\
& +\frac{r z}{|z|} \int_{0}^{\pi} \sin \theta \tan ^{-1}\left[\frac{(1-r \cos \theta)|z|}{r \sin \theta F}\right] d \theta \\
& \left.+\frac{\pi}{2}(1-r+|1-r|)\right\},
\end{aligned}
$$

where $F=\left(z^{2}+r^{2}+1-2 r \cos \theta\right)^{3}$.

These expressions were integrated by a computer. The second integral in $h_{r}(r, z)$ is improper for $z=0$; consequently, for $z=0$ it was integrated by parts analytically to avoid computer difficulties.

Five-place tables (to be published) of $h_{z}(r, z)$ and $h_{r}(r, z)$ were automatically printed by the computer and Figs. 3 and 4 were plotted from the tables. 